\title{
Instituições psiquiátricas e comunidades: um estudo de demanda em saúde mental no Estado de São Paulo, Brasil 1
}

\author{
Psychiatric institutions and communities: \\ a study of the demand for mental health care \\ in the State of São Paulo, Brazil 1
}

Selma Lancman 2

\footnotetext{
1 Pesquisa financiada pelo CNPq, processo № 410106/90.

2 Departamento de Clínica Médica, Faculdade de Medicina, Universidade de São Paulo. Rua Cipotânea 51, Cidade Universitária Armando Sales de Oliveira, São Paulo, SP 05360-000, Brasil.
}

Abstract Psychiatric hospitals have been accused of being both ineffective institutions and the main parties responsible for the chronic status of the mentally ill. However, there is a lack of research on the influence of mental health care facilities on either the communities in which they are located or the nearby out-patient services. Their presence may be a cause for increased psychiatric demand. This study investigates a possible association between the existence of such hospitals in given communities and an increase in hospitalization and demand for primary care services. The author compares the demand and client profile of out-patient services in similar communities, both with and without psychiatric hospitals Some evidence was found that in communities with such hospital s there is an increase in both hospital admittance and consultations in mental health care.

Key words Mental Health Services; Psychiatric Reform; Mental Health; Health Evaluation

Resumo Os hospitais psi quiátri cos têm si do freqüentemente denunciados como espaços ineficazes e são apontados como um dos principais responsáveis pel a cronificação dos doentes mentais. Faltam, porém, estudos que verifi quem a influência destes asilos sobre as comunidades que os abrigam e sobre os servi ços extra-hospitalares próximos a eles, podendo ser sua presença mais um fator gerador de demanda psi quiátrica. Esta pesquisa pretende verificar a associação entrea existência destes hospi tais em dadas comuni dades e o aumento de internações e de demanda aos serviços de atenção primária em saúde. Para tanto, procuramos verificar se a existência destes hospitais poderia estar modificando o perfil da clientela atendida, comparando a demanda que procurava os serviços extramanicomiais em cidades semel hantes que tivessem e que não tivessem hospitais psi quiátri cos. Foram encontradas al gu mas evidências de que, nas cidades com hospi tais, houve um aumento expressivo não somente das internações, mas também do número de consultas em saúde mental.

Palavras-chave Serviços de Saúde Mental; Reforma Psiquiátrica; Saúde Mental; Avaliação em Saúde 


\section{Introdução}

Vários estudos nas últimas décadas vêm apontando para a necessidade de avanços nas práticas em saúde mental, devido ao reconhecimento da falência do modelo de atenção centrado nos hospitais psiquiátricos, da pouca eficácia destas internações, dos seus efeitos cronificadores sobre os internados e às freqüentes denúncias da violação dos direitos humanos dos pacientes nestas instituições. Entre eles, destacamos os trabalhos de Amarante (1994), Giordano (1989), Lancman (1988), Lougon (1987), Moreira (1983), Sampaio (1989) e Urquiza (1991).

A necessidade de criação de novos modelos alternativos e a constituição de uma nova rede de atendimento em saúde mental que pudesse, gradativamente, substituir os hospitais desencadeou a implantação de um conjunto de serviços ambulatoriais e de atenção primária, principalmente a partir da década de 80 . Esses serviços visavam a remanejar os gastos com a assistência psiquiátrica hospitalar, para o desenvolvimento de uma rede extramanicomial eficiente que humanizasse $o$ atendimento oferecido (Portocarrero, 1990).

Essa mudança no enfoque da atenção psiquiátrica - das internações para os serviços de atenção primária e secundária - fez com que a incidência de internações se constituísse num parâmetro importante em psiquiatria, tendendo a ganhar relevância nos trabal hos de avaliação de qualidade. Mas, a despeito de sua força enquanto indicador, quando analisado de forma isolada não tem qualquer valor preditivo de boa ou má assistência.

Há uma carência de indicadores nessa área, reflexo da pouca utilização da epidemiologia em saúde mental. Isso ocorre por vários motivos, dentre eles a dificuldade de transposição dos instrumentos da epidemiologia para essa área e pela dificuldade de se definir e precisar vários aspectos das doenças mentais (Almeida Filho, 1989). Os parâmetros comumente utilizados na assistência médica não se adaptam, por vezes, à realidade dos serviços de saúde mental (Bastos \& Castiel, 1994). Os diagnósticos psiquiátricos são de baixa confiabilidade, quer pela falta de um esquema teórico passível de comprovação laboratorial e universal, quer pela forma rápida e superficial com que muitas muitas vezes são realizados. Além disso, temse um universo extremamente amplo e detaIhado de diagnósticos para uma gama muito reduzida de terapêuticas (Sarraceno et al., 1994). Estabelecer a prevalência das doenças mentais é uma tarefa complexa: dificuldade de se definir o seu início e fim; queixas muitas vezes indistinguíveis das problemáticas pessoais enfrentadas num cotidiano permeado por crises sócio-ecônomicas, instabilidade social etc.; além da "medicalização" excessiva da população, expressa pela manutenção em atendimento de pacientes que poderiam ser gradativamente desligados dos serviços (Almeida Filho, 1987).

Por outro lado, os debates e estudos sobre avaliação em saúde mental, ao enfocarem as mudanças no modelo de assistência e denunciarem os efeitos negativos dos asilamentos, não engl obaram a influência que estas instituições poderiam estar exercendo não somente sobre os internados, mas também sobre a população que freqüenta os demais serviços de atendimento à saúde mental e a comunidade em geral, tornando-os mais um fator gerador de demanda.

A existência de hospitais psiquiátricos em determinadas cidades e o tipo de convivência estabelecida entre o hospital e a comunidade, ao longo do tempo, podem estar associados a um processo de "medicalização" e refletir-se num aumento da demanda psiquiátrica, verificada através de maior freqüência de internações e de consultas ambulatoriais, pois estaria criado um "hábito" dentro das comunidades circunvizinhas aos asilos de resolver seus problemas "psiquiatrizando-os" (Lancman, 1995).

Estamos empregando o termo "psiquiatrização" a exemplo do conceito de "medicalização", utilizado por Donnangelo \& Pereira (1979:p.33), quando analisa a generalização no consumo dos serviços de saúde, através do processo de "...ampliação quantitativa e a incorporação crescente da população aos cuidados médicos e, como segundo aspecto, a extensão do campo da normatividade da medicina por referência às representações ou concepções de saúde e dos meios para se obtê-la, bem como às condições gerais de vida".

Esclarecemos que o conceito de "medicalização" e, por decorrência, o de "psiquiatrização" é mais complexo do que a extensão de cobertura e incorporação da população aos cuidados médicos, pois alude a processos sóciohistóricos específicos. No nosso caso, o processo de "psiquiatrização" ocorreria pela facilidade de acesso da população aos leitos psiquiátricos e serviços extra-hospitalares, pela facilidade na obtenção de psicofármacos e, principalmente, pela influência cultural do hospital sobre as comunidades aliada à dificuldade de se precisar e diferenciar os diagnósticos psiquiátricos das problemáticas sociais geradas no cotidiano das pessoas. 
Alguns dados sobre a assistência à saúde mental no Brasil e no Estado de São Paulo

No Brasil, em 1990, existiam 95.506 leitos psiquiátricos, divididos num total de 375 hospitais (MS, 1989). Destes, $26,2 \%$ eram leitos públicos e $73,8 \%$ privados, na sua maior parte conveniados com a rede pública. $\mathrm{O}$ tempo médio de permanência nos hospitais era de 69,7 dias, havendo uma relação de 0,66 leitos/ 1.000 habitantes. As internações psiquiátricas, por estes mesmos dados, representavam a segunda fonte de despesa com internações hospitalares no país, só perdendo para as doenças respiratórias (Savio et al., 1994). Em 1993, esse número baixou para 313 hospitais vinculados ao SUS, que ofereciam 93.000 leitos (MS, 1993). O tempo médio de internação para o País também caiu para 55,6 dias. Esta redução de leitos psiquiátricos parece refletir uma nova política de se evitar o confinamento dos doentes mentais, mas também poderia significar uma queda na qualidade do atendimento (Seidl et al., 1990).

O Ministério da Saúde trabalha com uma prevalência psiquiátrica de $13 \%$ sobre o total da população, embora reconheça que há um distanciamento entre doença percebida e doença diagnosticada, e estabelece como limite máximo aceitável a existência de 0,5 leito/ 1.000 habitantes (MS, 1991).

No Estado de São Paulo, existiam, em 1989, 116 hospitais psiquiátricos, sendo que 39 estavam localizados na Grande São Paulo e o restante no interior. Deste total, 11 eram estaduais e os 105 restantes particulares, apesar de manterem, na sua maioria, convênios com a Previdência Social e com a Secretaria da Saúde do Estado. No início da década de 80, esses hospitais ofereciam 39.112 leitos, número que caiu para 34.000 em 1990. No Estado de São Paulo, estão $36,5 \%$ dos leitos psiquiátricos do País, numa proporção de 1,28 leitos/ 1.000 habitantes (SES, 1981).

Em São Paulo, a redução no número de leitos psiquiátricos está associada a uma nova política de saúde mental, iniciada na década de 80, que passou a investir em assistência extra-hospitalar, com a criação de ambulatórios e de outros serviços de Saúde Mental na rede básica. Pretendia, através deles, racionalizar as internações e oferecer outro tipo de atendimento que não o hospitalar, além de controlar a porta de entrada dos asilos, diminuindo assim o número de internações e reinternações (Pitta-Hoisel, 1984).

Um maior número de atendimentos ambulatoriais não significa necessariamente uma maior resolutividade. Ao contrário, sabe-se que muitas vezes esses serviços são de pouca eficácia, geradores de demanda e cronificadores, faltando estudos que mostrem o destino da demanda que passa a freqüentar os ambulatórios de saúde mental.

\section{Aspectos metodológicos}

Neste estudo, procuramos verificar, por meio da freqüência tanto de internação como de consultas e altas ambulatoriais, se a existência de serviços de saúde mental e, em particular, dos hospitais psiquiátricos poderia estar modificando o perfil da clientela atendida, verificado através. Para tanto, optamos por trabal har com dados de produção de serviços psiquiátricos ambulatoriais e de emergência, comparando cidades semelhantes que tivessem e que não tivessem hospitais psiquiátricos.

Escolhemos quatro cidades, duas no interior do Estado de São Paulo - São Carlos e Araraquara - e duas periféricas à capital - Ferraz deVasconcelos e Franco da Rocha. Para a escoIha destas cidades, levamos em conta semeIhanças em termos demográficos e sócio-econômicos, bem como a distância e acesso em relação à capital .

Nessas cidades, pudemos levantar dados sobre todos os pacientes que procuram atendimento psiquiátrico no período estudado, mediante dados de prontuários e entrevistas.

Essas informações permitiram traçar não só um perfil da demanda psiquátrica nesses municípios, como também estabelecer comparações, detectando especificidades e semeIhanças entre os vários serviços e regiões.

As cidades de São Carlos e Araraquara distam cerca de $250 \mathrm{~km}$ da capital e constituem grandes pólos econômicos do interior paulista. Possuíam, em 1990, segundo estimativas da Fundação Seade, uma média de 160.000 habitantes. Além da tradição agrícola, os dois municípios possuem vastos parques industriais e cerca de $40 \%$ da população ganha mais de cinco salários mínimos. As duas cidades são também tradicionais pólos universitários, com escolas públicas e particulares de ensino superior. Suas redes de esgoto e água cobrem mais de $95 \%$ da população. Apresentavam, em 1987, respectivamente, coeficientes de mortalidade infantil de 28,33 e 23,7/ 1.000 nascidos vivos.

São Carlos possuía, em 1990, 11 Postos de Assistência Médica em bai rros periféricos, um Pronto Socorro Municipal, uma Santa Casa de Misericórdia e um Centro de Saúde de maior complexidade com uma equipe de saúde men- 
tal e, quando necessário, utilizava, como referência, o hospital psiquiátrico de Santa Rita do Passa Quatro, que funcionava com 1.056 leitos operacionais.

Em Araraquara, havia seis hospitais (sendo dois psiquiátricos), com uma média de 5,2 leitos/ 1.000 hab. em hospitais gerais e 2,39 leitos/ 1.000 nos psiquiátricos. Existiam ainda nove Unidades de Saúde (localizadas em bairros periféricos, prestando atenção primária), um Posto de Assistência Médica e um Centro de Saúde Escola (Cesa), serviço de extensão vinculado à Faculdade de Saúde Pública da USP. Os dois últimos atendiam casos de maior complexidade e prestavam atendimentos psiquiátricos.

A cidade de Ferraz de Vasconcelos está situada na periferia, a $18 \mathrm{~km}$ da capital paulista. Em 1990, tinha 87.892 habitantes e um coeficiente de mortalidade infantil de 42/ 1.000. Pertencia ao Escritório Regional de Saúde-13 (Ersa 13), que tinha como sede Mogi das Cruzes, e congregava também as cidades de Suzano, Itaquaquecetuba, Guararema, Santa Isabel e Poá. O Município possuía oito Unidades Básicas de Saúde, um Ambulatório de Especialidades e um Hospital Regional com 248 leitos, onde funcionava um serviço de emergência psiquiátrica. Em relação à saúde mental, a cidade não possuía atendimento em nível primário, utilizando como referência o Ambulatório de Saúde Mental de Mogi das Cruzes e, para as internações, os hospitais de Itaquaquecetuba, com 584 leitos. Vale a pena esclarecer que, com a reforma administrativa ocorrida na Secretaria Estadual de Saúde, em São Paulo, na década de 80 , todos os serviços de saúde foram reagrupados, passando a ser gerenciados de forma descentralizada por escritórios regionais que ficaram conhecidos como ERSA e numerados segundo a região a que pertenciam.

Assim como o Município de Franco da Rocha, Ferraz de Vasconcelos possuía um pequeno parque industrial, era servida por rede ferroviária e caracterizava-se por ser cidade-dor- mitório, abrigando diversos trabal hadores da região metropolitana, que tinham nos trens um meio rápido e barato de transporte.

Franco da Rocha tinha, em 1990, um total de 52.669 habitantes. Pertencia ao Escritório Regional de Saúde-14, que congregava ainda as cidades de Cai eiras, Cajamar, Francisco Morato e Mairiporã. Possuía um Centro de Saúde, uma Unidade Básica, um Ambulatório de Saúde Mental e um serviço de emergência psiquiátrica chamado Pré-Internação - estes dois últimos estavam localizados nas dependências do Juqueri, tradicional hospital psiquiátrico paulistano, que na época da pesquisa, apesar de não receber novas internações, ainda possuía 2.000 pacientes crônicos e até hoje é considerado um marco na história daquele município. As internações mais prolongadas eram encaminhadas para o hospital de Mairiporã.

Os dois serviços de emergência, em Franco da Rocha e em Ferraz de Vasconcelos, foram concebidos para atender somente casos graves, e ficavam abertos 24 horas por dia, em sistema de plantão. Pelas dificuldades de acesso, agenda e horário de funcionamento dos demais serviços, eles terminavam por se caracterizar como ambulatórios e atender boa parte da demanda psiquiátrica dos dois municípios. Ambos possuíam pequenas enfermarias para internações de curta permanência. A Pré-Internação de Franco da Rocha e o Pronto-atendimento de Ferraz de Vasconcelos atendiam, respectivamente, $51,3 \%$ e $48,1 \%$ de toda a demanda de cada região (Tabela 1 ).

Realizamos dois tipos de levantamento: um em prontuários e outro através de entrevistas com pacientes na fila de espera. Entrevistamos $63,4 \%$ dos pacientes atendidos em São Carlos, $66,4 \%$ em Araraquara, 46,5\% em Franco da Rocha e 29,2\% em Ferraz de Vasconcelos. Como há grande diferença entre o número de consultas e o número de pacientes consultados, nas nossas análises cada paciente foi contabilizado uma única vez, independente do número de vezes que procurou o serviço.

Tabela 1

N úmero de pacientes das amostras estudadas em São Carlos, Araraquara, F. da Rocha e F. de Vasconcelos, out-nov, 1990.

\begin{tabular}{lcccccc}
\hline & S. Carlos & Araraquara & \multicolumn{2}{c}{ F. Rocha } & \multicolumn{2}{c}{ F. Vasconcelos } \\
& & & & Pré-Inter & Ersa 14 & Pronto Atend. Ersa 13 \\
\hline Consultas registradas & 791 & 1.210 & 1.338 & 2.675 & 1.270 & 2.638 \\
Pacientes registrados & 675 & 987 & 694 & 1.140 & 983 & 2.156 \\
Pacientes entrevistados & 428 & 656 & 323 & & 288 \\
\hline
\end{tabular}


Por intermédio dos prontuários, foi possível abranger o total de consultas em saúde mental realizadas no período e coletar dados gerais, tais como: nome, idade, sexo, diagnóstico e encaminhamento. Mediante os questionários foram obtidos outros dados, a saber: tempo de tratamento psiquiátrico e internações anteriores. Em São Carlos e Araraquara, foi possível cobrir todos os serviços devido à facilidade de acesso e ao reduzi do horário de funcionamento do Cesa. Em Franco da Rocha e Ferraz de Vasconcelos, as entrevistas foram realizadas nas emergências psiquiátricas, locais que concentram a maior demanda. O levantamento de prontuários abrangeu todos os ambulatórios, inclusive o de Mogi das Cruzes (referência para Ferraz de Vasconcelos).

Alguns agrupamentos tiveram que ser feitos. Reunimos os diagnósticos em grupos mais gerais, de acordo com a Classificação Internacional de Doenças (CID - 9ạ revisão, 1979). Juntamos à categoria "outros" os diagnósticos que apareceram com freqüência reduzida, tais como: oligofrenias, uso de drogas, epilepsias e psicoses orgânicas.

Os encaminhamentos também foram agrupados por critérios mais globais. A categoria "outros" refere-se aos encaminhamentos para médicos especialistas de outras áreas, orientações, encaminhamentos para consultas com equipe multiprofissional não médica e internação em hospital não psiquiátrico. Na categoria "retorno", incluímos os pacientes que receberam como encaminhamento "medicação".

\section{Perfil comparativo da demanda} psiquiátrica nos municípios pesquisados

Apresentamos, a seguir, um panorama comparativo da demanda psiquiátrica nas cidades pesquisadas, em relação às principais variáveis estudadas: idade, sexo, diagnóstico, encaminhamento, tempo de tratamento em psiquiatria e internações anteriores em hospitais psiquiátricos.

A distribuição dos habitantes por faixa etária nas cidades, segundo estimativas da Fundação Seade para 1990, dá-se do seguinte modo: as quatro cidades possuem uma população jovem, com predomínio de pessoas com menos de vinte anos, exceto Araraquara, cuja população de vinte a quarenta anos ultrapassa a daqueles com menos vinte anos. Este percentual de jovens é maior nas cidades de Franco da Rocha e Ferraz de Vasconcelos. Observamos, também, que Araraquara e São Carlos possuem uma população mais idosa ( $10 \%$ com mais de sessenta anos, enquanto nos outros municípios esse percentual era de $5 \%$ ).

Com relação à idade da população atendida nos serviços de saúde mental, encontramos (Figura 1), nos municípios estudados, uma maior concentração de pacientes na faixa de vinte a quarenta anos (em especial em Franco da Rocha, onde esta proporção é maior), seguida da faixa de quarenta a sessenta anos. Ferraz de Vasconcelos, apesar de apresentar menor proporção de idosos entre seus moradores (apenas 4\% têm mais de sessenta anos), é a cidade com a maior concentração de pacientes nessa faixa, ultrapassando até o número de pacientes na faixa de quarenta a sessenta anos.

Apesar de as quatro cidades apresentarem uma distribuição quase eqüitativa entre homens e mulheres, com uma pequena predominância feminina em Franco da Rocha, na nossa amostra, verificamos uma procura psiquiátrica maior entre mulheres nas cidades de Araraquara e São Carlos (60\% em ambas), e de homens, em Franco da Rocha e Ferraz de Vasconcelos (em média 55\%). No entanto, esperávamos uma maior proporção de homens procurando as emergências psiquiátricas, devido ao tipo de serviço pesquisado, do horário de funcionamento e à proporção de alcoolistas que em geral são homens e buscam, com muito mais freqüência, os pronto-atendimentos.

Ao estudarmos a distribuição dos diagnósticos na Figura 2, observamos um predomínio de psicoses em todas as cidades, exceto em São Carlos, onde foram diagnosticadas mais neuroses. Entre Ferraz de Vasconcelos e Franco da

Figura 1

Distribuição da demanda psiquiátrica segundo a idade em Araraquara, S. Carlos, F. Vasconcelos, F. Rocha, out-nov, 1990.

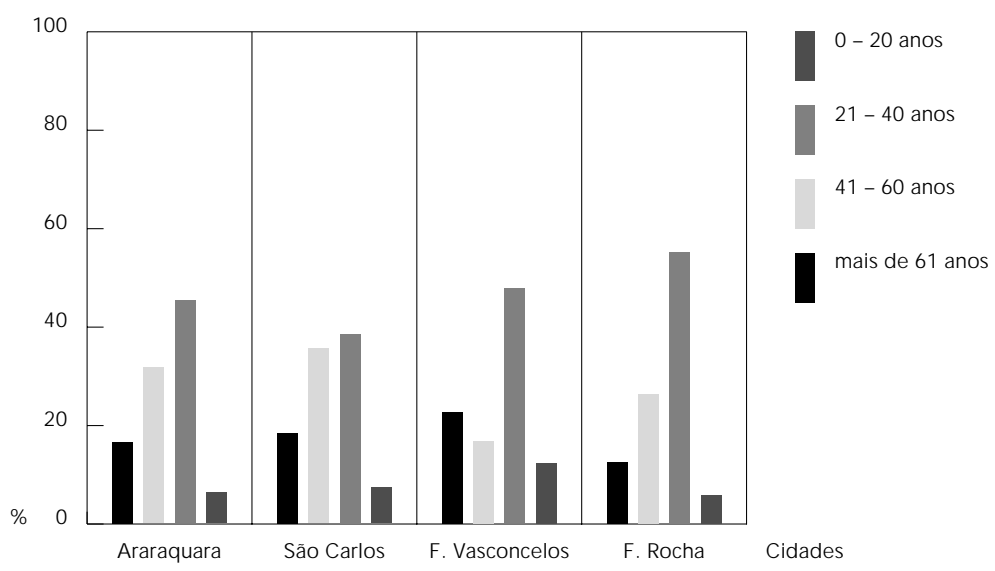

OBS: Incluímos somente os pacientes residentes na cidade onde foram atendidos. 
Figura 2

Distribuição dos pacientes atendidos segundo o diagnóstico recebido em Araraquara, S. Carlos, F. Vasconcelos, F. Rocha, out-nov, 1990.

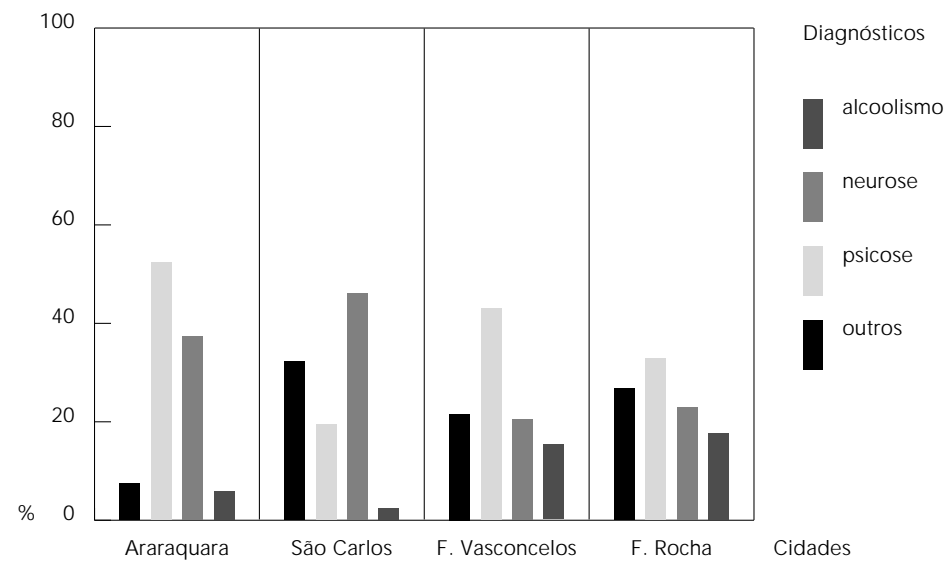

Figura 3

Distribuição dos pacientes atendidos segundo os encaminhamentos recebidos em Araraquara, S. Carlos, F. Vasconcelos, F. Rocha, out-nov, 1990.

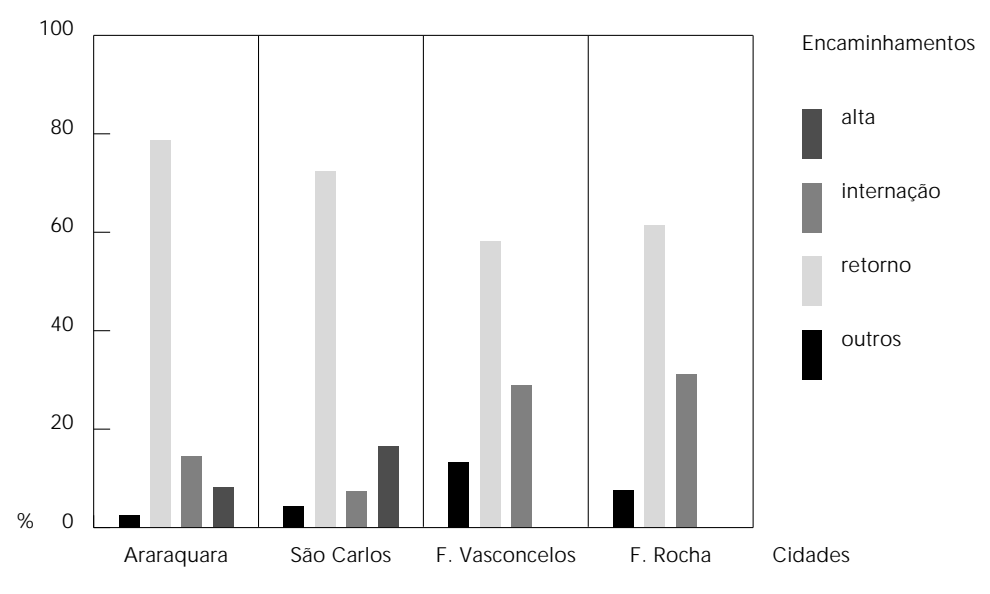

Rocha, a distribuição dos diagnósticos é semeIhante, com certo aumento na proporção de psicoses registradas em Ferraz de Vasconcelos, o que ocorre pela diferença entre os diagnósticos de alcoolismo e de neuroses. Mas o que nos chama a atenção é a grande diferença na proporção de psicoses diagnosticadas entre Araraquara e São Carlos, 51,4\% e 19,3\% respectivamente, enquanto os percentuais de neuroses diagnosticadas se invertem a favor desse último município. Isso significa que mais da metade dos pacientes consultados em Araraquara recebeu diagnóstico de psicose. Já o alcoolismo está mais presente em Franco da Rocha e Ferraz de Vasconcelos. A percentagem de diag- nósticos de neurose entre as mulheres é bem mais alta em todos os serviços. Em relação às psicoses, encontramos uma freqüência maior de homens em todas as cidades, embora em Franco da Rocha o número se aproximasse ao das mulheres.

Na Figura 3, analisamos os encaminhamentos prescritos. Observamos a ausência de altas em Ferraz de Vasconcelos e Franco da Rocha, enquanto em São Carlos e Araraquara encontramos as percentagens, respectivamente, de $16,4 \%$ e $7,0 \%$ de altas. Esse dado está relacionado com o elevado número de pacientes que foram encaminhados para retorno no mesmo serviço, ou seja, a maior parte dos pacientes freqüenta os serviços por muito tempo e as emergências terminam funcionando, em parte, como ambulatórios. A ausência de al tas em Ferraz de Vasconcelos e Franco da Rocha devese principalmente à natureza do atendimento nas emergências, onde em geral se interna ou se encaminha a outros serviços.

A proporção de internações psiquiátricas em Ferraz de Vasconcelos e Franco da Rocha é muito próxima (perto de 30\%) e bem maior do que nas outras duas cidades. Chama-nos a atenção a percentagem de internações ocorridas em Araraquara (13,0\%), que é alta para serviços ambulatoriais, principalmente quando comparada à de São Carlos (7,1\%).

O número el evado de internações em Araraquara está relacionado com a elevada percentagem de diagnósticos de psicose verificada anteriormente e, também, com a maior proporção de internações entre pacientes com esse diagnóstico. O número de internações entre alcoolistas, em Araraquara, também é maior do que em São Carlos.

Encontramos, como era de se esperar, uma percentagem maior de internações nas emergências, principalmente entre psicóticos e alcoolistas. Isso se deve à natureza desses serviços, que atendem prioritariamente pacientes em surto, enquanto os ambulatórios mantêm atendimento, preferencialmente, a pacientes crônicos, mesmo fora dos períodos de crise.

A distribuição dos encaminhamentos por sexo revela um predomínio de homens internados em todas as localidades, até mesmo em Araraquara e São Carlos, onde as mulheres procuram mais os serviços. Ressalta-se que, nestas duas cidades, a diferença é mais marcante (respectivamente, $24,3 \%$ homens para $6,2 \%$ mulheres e $12,4 \%$ para $3,1 \%$ ) do que em Ferraz deVasconcelos (34,7\% para $21,1 \%$ ) e Franco da Rocha (34,6\% para $22,9 \%)$.

Já entre os encaminhamentos para retorno, há um predomínio das mulheres em todas as 
Percentual dos diagnósticos prescritos nos serviços de saúde mental pesquisados, em relação ao sexo dos pacientes, em Araraquara, São Carlos, Franco da Rocha e Ferraz de Vasconcelos, out-nov, 1990.

\begin{tabular}{lrrrrrrrr}
\hline \multirow{2}{*}{ Diagnóstico } & \multicolumn{2}{c}{ S. Carlos } & \multicolumn{2}{c}{ Araraquara } & \multicolumn{2}{c}{ F. Rocha } & \multicolumn{2}{c}{$\begin{array}{c}\text { F. Vasconcelos } \\
H\end{array}$} \\
& $H$ & M & H & M & H & M & & \\
\hline Alcoolismo & 5,0 & 0,3 & 9,6 & 1,6 & 30,6 & 2,9 & 23,6 & 3,6 \\
Neurose & 30,3 & 57,5 & 21,9 & 45,8 & 12,6 & 38,1 & 11,6 & 32,3 \\
Psicose & 23,4 & 16,4 & 56,8 & 47,7 & 33,3 & 31,0 & 45,8 & 39,2 \\
Outras & 41,3 & 25,8 & 11,7 & 5,0 & 23,4 & 28,0 & 19,0 & 25,1 \\
Total & 100,0 & 100,0 & 100,0 & 100,0 & 100,0 & 100,0 & 100,0 & 100,0 \\
\hline
\end{tabular}

Tabela 3

Número e percentagem de casos com diagnóstico de psicose, segundo o encaminhamento recebido nos serviços de saúde mental pesquisados em Araraquara, São Carlos, F. Rocha e F. Vasconcelos, out-nov, 1990.

\begin{tabular}{|c|c|c|c|c|c|c|c|c|}
\hline \multirow[t]{2}{*}{ Encaminhamentos } & \multicolumn{2}{|c|}{ Araraquara } & \multicolumn{2}{|c|}{ S. Carlos } & \multicolumn{2}{|c|}{ F. Rocha } & \multicolumn{2}{|c|}{ F. Vasconcelos } \\
\hline & $\mathrm{n}$ & $\%$ & $\mathrm{n}$ & $\%$ & $\mathrm{n}$ & $\%$ & $\mathrm{n}$ & $\%$ \\
\hline Alta & 15 & 10,2 & 5 & 5,6 & - & - & - & - \\
\hline Internação & 41 & 27,9 & 12 & 13,5 & 101 & 53,7 & 175 & 48,7 \\
\hline Retorno & 187 & 59,2 & 71 & 79,8 & 67 & 35,6 & 163 & 45,4 \\
\hline Outros & 4 & 2,7 & 1 & 1,1 & 20 & 10,6 & 21 & 5,8 \\
\hline Total & 247 & 100,0 & 89 & 100,0 & 188 & 100,0 & 359 & 100,0 \\
\hline
\end{tabular}

cidades pesquisadas. Entre os que tiveram alta em Araraquara e São Carlos, não há diferença significativa em relação ao sexo.

Na distribuição do sexo, segundo o diagnóstico recebido, verificamos, na Tabela 2, um predomínio muito acentuado de alcoolismo entre os homens e de neuroses entre as mulheres em todos os municípios pesquisados. Entre os psicóticos, embora predominem os homens, a diferença é menos acentuada do que nos dois diagnósticos anteriores.

Quando analisamos os encaminhamentos em relação aos diagnósticos, percebemos, na Tabela 3, que, dentre os pacientes diagnosticados com psicose, aproximadamente $50 \%$ das pessoas atendidas em Ferraz de Vasconcelos e em Franco da Rocha receberam como encaminhamento a internação. Esse percentual é bem mais elevado do que o de Araraquara e o de São Carlos. Entre as cidades pareadas, com ou sem hospital psiquiátrico, não se encontram diferenças importantes no que concerne a Ferraz de Vasconcelos e Franco da Rocha. Porém, quando analisamos as diferenças entre São Carlos e Araraquara, percebemos que esta última interna mais seus psicóticos e dá mais altas, enquanto São Carlos agenda mais retornos de pacientes com esse diagnóstico. A ausência de diferenças entre Ferraz de Vasconcelos e Franco da Rocha parece contrariar nossa hipótese inicial: esperávamos encontrar mais internações no segundo município, devido à presença do hospital.

Ao estudarmos o tempo do tratamento, encontramos um maior número de pacientes freqüentando os serviços há mais de cinco anos, em todas as cidades (em média 40\%).

Em relação à ocorrência prévia de internações psiquiátricas (Tabela 4), observamos percentuais semelhantes em Franco da Rocha e em Ferraz de Vasconcelos (55\% de egressos). Chamou-nos a atenção a diferença entre São Carlos e Araraquara, com uma maior quantidade de pessoas, nessa última cidade, egressas de hospitais (35,9\% e 48,3\% respectivamente).

Encontramos em Ferraz de Vasconcelos, Franco da Rocha e São Carlos uma percentagem média de $79 \%$ de egressos entre os psicóticos, enquanto em Araraquara uma proporção menor, de 65,2\%. Em contrapartida, Araraquara foi a cidade com maior percentagem de internações prévias entre al coolistas (74\%), enquanto em Ferraz de Vasconcelos e em Franco da Rocha esse percentual era em média de $68 \%$, e em São Carlos de 54\%.

Outro dado que nos chamou a atenção foi o número de pessoas diagnosticadas como neu- 
Tabela 4

Número e distribuição percentual dos pacientes segundo os diagnósticos recebidos e que já haviam passado por internações anteriores nos serviços de saúde mental pesquisados, em Araraquara, S. Carlos, F. Rocha e F. Vasconcelos, out-nov, 1990.

\begin{tabular}{|c|c|c|c|c|c|c|c|c|}
\hline \multirow[t]{2}{*}{ Diagnósticos } & \multicolumn{2}{|c|}{ Araraquara } & \multicolumn{2}{|c|}{ S.Carlos } & \multicolumn{2}{|c|}{ F.Rocha } & \multicolumn{2}{|c|}{ F.Vasconcelos } \\
\hline & $\mathrm{S}$ & $\mathrm{n}$ & $\mathrm{S}$ & $\mathrm{n}$ & $\mathrm{S}$ & $\mathrm{n}$ & $\mathrm{S}$ & $n$ \\
\hline Alcoolismo & 74,2 & 31 & 54,5 & 11 & 67,5 & 40 & 69,7 & 33 \\
\hline Neurose & 20,9 & 206 & 11,8 & 127 & 29,9 & 77 & 32,4 & 37 \\
\hline Psicose & 65,2 & 250 & 79,4 & 64 & 82,0 & 95 & 79,1 & 115 \\
\hline Outros & 64,7 & 51 & 20,0 & 60 & 33,3 & 377 & 35,0 & 60 \\
\hline
\end{tabular}

róticas e que já haviam passado por internações psiquiátricas. Em Ferraz de Vasconcelos esse número era de 32,4\%; em Franco da Rocha, de 29,9\%; em Araraquara, 21\% e em São Carlos, 11,8\%.

\section{Considerações finais}

Frente ao exposto, apresentamos al gumas considerações, dentro dos limites que os dados permitem concluir, sem pretender que possam dar conta de toda a complexi dade dos aspectos da demanda psiquiátrica estudada.

Nosso resultados estão em acordo com um estudo feito no Departamento de Neurologia e Psiquiatria da Faculdade de Medicina de Botucatu da Unesp (Torres et. al., 1989), em que as autoras, ao verificarem, através de prontuários, a demanda que procura o ambulatório de psiquiatria, encontraram uma concentração maior de mulheres em todas as faixas etárias, além de um predomínio de adultos jovens.

A precariedade dos registros nos prontuários e fichas, assim como a ausência de estatísticas de produtividade nos serviços, dificultaram os estudos e as comparações, permitindonos apontar apenas algumas tendências em relação aos dados por nós estudados.

Encontramos, em todos os locais pesquisados, uma quase ausência de altas, um excessivo tempo de utilização dos serviços e um elevado número de internações e reinternações.

As diferenças observadas sugerem uma indefinição diagnóstica e uma subjetividade nas avaliações das doenças psiquiátricas. Isto está relacionado, entre outros fatores, a concepções teóricas e escolas psiquiátricas diferentes e à dificuldade de comprovação laboratorial e de universalização das observações. Nossa constatação está de acordo com um estudo realizado em três hospitais psiquiátricos no Rio de Janeiro. Em duas situações diferentes, foi encon- trada, para o mesmo paciente, uma discordância diagnóstica em internações psiquiátricas diversas, dependendo do médico que efetuou $o$ atendimento (MS, 1989).

Nossa hipótese inicial quanto à maior freqüência de internações nas cidades possuidoras de hospital psiquiátrico não pôde ser plenamente respondida. Encontramos dados de internação parecidos em Franco da Rocha e Ferraz de Vasconcelos. Por outro lado, em Araraquara ocorreu um acréscimo significativo no número de internações e reinternações, em relação a São Carlos.

Frente à constatação exposta, comparamos nossos resultados com o número de consultas e internações sugeridos pelo Ministério da Saúde para a Região Sudeste e para o Estado de São Paulo. Estimamos os dados para todo o ano e os relacionamos com o total da população de cada município. Para calcular o número de consultas entre os moradores, mesmo sabendo dos riscos de possíveis vieses pelo dados perdidos, fizemos uma projeção, aplicando o percentual de retornos que havíamos encontrado anteriormente: $86 \%$ em São Carlos, $81 \%$ em Araraquara, 51\% em Franco da Rocha e $31 \%$ em Ferraz de Vasconcelos. Lembramos que este percentual foi calculado sobre o total de pacientes atendidos, em cujos prontuários foi possível encontrar esta informação. Em Ferraz de Vasconcelos, tivemos que excluir da nossa amostra os pacientes oriundos dessa cidade e atendidos em Mogi das Cruzes, por não dispormos dessa informação. Em Franco da Rocha, partimos do total de pacientes atendidos em saúde mental, em todos os serviços (Tabela 5).

Através dessa Tabela, podemos concluir que a cobertura para as consultas é, em todas as cidades pesquisadas, bem maior do que aquela apontada pelo Ministério da Saúde, superando em muito o número esperado para o País (23/ 1.000 hab.) 
Estimativa da produção de serviços psiquiátricos, consultas e internações ao ano - cobertura populacional/1.000 hab., em Araraquara, S. Carlos, F. Rocha e F. Vasconcelos, 1990.

\begin{tabular}{lcccc}
\hline Cidades & Araraquara & S. Carlos & F. Rocha & F. Vasconcelos \\
\hline Cons./Ano & 9.680 & 6.328 & 15.936 & 10.160 \\
Cons. entre moradores & 7.840 & 5.442 & 3.127 & 3.149 \\
Cons./1.000 hab. & 50,1 & 360 & 154,3 & 35,8 \\
Intern./Ano & 888 & 309 & 2.008 & 2.624 \\
Intern. entre moradores & 719 & 1,9 & 1.024 & 813 \\
Intern./1.000 hab. & 4,5 & 19,4 & 9,2 \\
\hline
\end{tabular}

Em relação às internações, encontramos em São Carlos números inferiores em relação aos sugeridos pelo Ministério para o Estado de São Paulo. Araraquara, Ferraz de Vasconcelos e Franco da Rocha apresentam número bem maiores. Esse indicador não especifica o tipo de serviço onde o atendimento foi realizado. Nas emergências, devido às suas características, é esperada uma freqüência de internações maior do que nos ambulatórios.

Quando comparamos cidades que possuem hospital psiquiátrico com cidades que não possuem, verificamos não só um acréscimo no número de internações, como também um aumento expressivo no número de consultas em saúde mental nos municípios com hospital.

Na Tabela 5, ao fazermos uma estimativa, em termos da cobertura assistencial em relação ao total de habitantes de cada uma das ci-

\section{Referências}

ALMEIDA FILHO, N., 1989. Epidemiologia sem Números: uma Introdução Crítica à Ciência Epidemiológica. Rio de Janeiro: Ed. Campus.

ALMEIDA FILHO, N., 1987. Epidemiologia social das desordens mentais: revisão da literatura latino americana, In: Cidadania eLoucura: Políticas de Saúde Mental no Brasil. (Almeida-Filho, org.), pp.103-131, Petrópolis: Ed. Vozes/Abrasco.

AM ARANTE, P., 1994. Asilos, alienistas: uma pequena História da Psiquiatria no Brasil. In: Psiquiatria Social eReforma Psiquiátrica. (Amarante, P., org.), pp: 73-81. Rio de Janeiro: Ed. Fiocruz.

BASTOS, F. I. P. \& CASTIEL, L. D., 1994. Epidemiologia e Saúde Mental no Campo Científico Contemporâneo: Labirintos que se entrecruzam? In: Psiquiatria Social e Reforma Psiquiátrica. (Amarante, P., org.), pp. 97-111. Rio de Janeiro: Ed. Fiocruz. dades, verificamos que a proporção de consultas em Araraquara é 47,3\%, maior que a de São Carlos. Em Franco da Rocha, esse coeficiente é $331 \%$ maior que em Ferraz de Vasconcelos.

Em relação ao número de internações, havíamos encontrado um percentual semelhante em Ferraz de Vasconcelos e Franco da Rocha frente ao total de atendimentos. No entanto, quando comparamos as hospitalizações em relação ao número de habitantes, em Franco da Rocha este percentual é $110 \%$ maior que o de Ferraz de Vasconcelos. Em Araraquara, ele é 133\% maior que em São Carlos.

Embora os dados apresentados não comprovem plenamente nossa hipótese quanto à influência dos hospitais psiquiátricos na geração de demanda nos serviços de saúde mental, encontramos uma associação positiva em favor dos nossos pressupostos, tanto em Araraquara, quanto em Franco da Rocha.
DONNANGELO, M. C. F. \& PEREIRA, L., 1979. Saúdee Sociedade, 2a ed. São Paulo: Ed. Duas Cidades.

GIORDANO, S. J., 1989. A Persistência da Higieneea Doença Mental: Contribuição à História das Políticas de Saúde Mental no Estado de São Paulo. Dissertação de Mestrado, São Paulo: Faculdade de Medicina, Universidade de São Paulo.

LANCM AN, S., 1988. A Loucura do Outro: o Juqueri no Discurso de seus Protagonistas. Dissertação de Mestrado, Salvador: Faculdade de Medicina, Universidade Federal da Bahia.

LANCM AN, S., 1995. Loucura e Espaço Urbano: Um Estudo Sobre as Rel ações Franco da Rocha - Juqueri. Tese de Doutorado, Campinas: Departamento de Psiquiatria e Psicologia Médica, Universidade de Campinas. 
LOUGON, M., 1987. Os Caminho da Mudança: Alienados, Alienistas e a Desinstitucionalização da Assistência Psiquiátrica Pública. Dissertação de Mestrado, Rio de Janeiro: Museu Nacional, Universidade Federal do Rio de Janeiro.

MS (Ministério da Saúde), 1991. Pesquisa de Morbidade em Seis Cidades Brasileiras em 1990, em População Acima de 14 Anos. São Paulo: Centro de Epidemiologia, Pesquisa e Informação (CEPI, SMS). (mimeo.)

MS (Ministério da Saúde), 1989. Informações para a Saúde, vol. 10, no 6, Brasília: Centro de Documentação.

MS (Ministério da Saúde), 1993. Reestruturação da Atenção em Saúde Mental: Situação Atual, Diretrizes e Estratégias. Brasília: Secretaria de Assistência à Saúde, Departamento de Assistência e Promoção à Saúde, Coordenação de Saúde Mental. (mimeo.)

MOREIRA, D., 1983. Psiquiatria: Controle e Repressão Social. Belo Horizonte: Ed. Vozes \& Fundação João Pinheiro.

PITTA-HOISEL, A. M., 1984. Sobre uma Política de Saúde Mental. Dissertação de Mestrado, São PauIo: Faculdade de Medicina, Universidade de São Paulo.

PORTOCARRERO, V. M., 1990. O Dispositivo da Saúde Mental: uma Metamorfose na Psiquiatria Brasileira. Tese de Doutorado, Rio de Janeiro: Instituto de Filosofia e Ciências Sociais, Universidade Federal do Rio Janeiro.
SAMPAIO, J. J. C., 1988. Hospital Psiquiátrico Público no Brasil: a Sobrevivência do Asilo e Outros Destinos Possíveis. Dissertação de Mestrado, Rio de Janeiro: Faculdade de Medicina, Universidade do Estado do Rio de Janeiro.

SARRACENO, B., ASIOLY, F., TOGNONI, G., 1994. Manual deSaúde Mental. São Paulo: Ed. Hucitec.

SAVIO, D. N. A., SEIDL, E.F., SCHECHTMAN, A., SILVA, R.C., 1994. Reestruturação da atenção em Saúde Mental: situação atual, diretrizes e estratégias. In: Psiquiatria Social e Reforma Psiquiátrica. (Amarante, P., org.), pp. 195-201. Rio de Janeiro: Ed. Fiocruz.

SEIDL, E. M. F. \& BRITO, E. C., 1990. Assistência à Saúde Mental no Brasil: Análise da Produção de Consultas e de Internações de 1984 a 1987. Brasília: Ministério da Saúde, Secretaria Nacional de Assistência à Saúde, Departamento Nacional de Programas de Saúde, Divisão Nacional de Saúde Mental. (mimeo.)

SES (Secretaria de Estado da Saúde), 1991. Plano de Atenção à Saúde Mental no Estado de São Paulo biênio 1991/92. São Paulo: SES. (mimeo.)

TORRES, A. R. \& CERQUEIRA, A. T. A. R, 1989. Avaliação Crítica de um Programa do Ambulatório de Psiquiatria de um Hospital Universitário. Botucatu: Departamento de Neurologia e Psiquiatria, Faculdade de Medicina, Universidade do Estado de São Paulo. (mimeo)

URQUIZA, L.1991. Um Tratamento para a Loucura: Contribuição à História da Emergência das Práticas Psiquiátricas no Estado de São Paulo. Dissertação de Mestrado, Campinas: Departamento de Psiquiatria e Psicologia Médica, Universidade de Campinas. 